\title{
miR612 is associated with esophageal squamous cell carcinoma development and metastasis, mediated through TP53
}

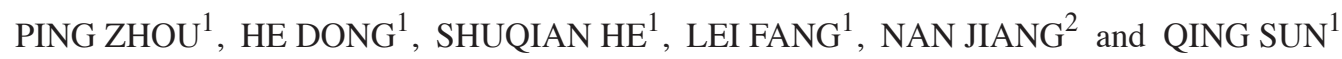 \\ ${ }^{1}$ Department of Pathology, Qianfoshan Hospital Affiliated with Shandong University; \\ ${ }^{2}$ Department of Pathology, Shandong University School of Medicine, Jinan, Shandong 250014, P.R. China
}

Received April 28, 2016; Accepted March 16, 2017

DOI: $10.3892 / \mathrm{mmr} .2017 .6808$

\begin{abstract}
MicroRNAs (miRNAs) serve an important role in the regulation of gene expression. In the present study, differential expressions of miRNAs were compared between esophageal squamous cell carcinoma (ESCC) tissues and normal esophageal tissues. In combination with miRNA target prediction databases, a significantly increased expression of miR-612 was discovered in ESCC. The relationship between miR-612 and TP53 gene expression and their roles in ESCC invasion and metastasis was further studied by reverse transcription-quantitative polymerase chain reaction and western blotting in EC109 cells and cancer tissues. The EC109 cell invasion and migration were significantly reduced after miR-612 expression was inhibited. The levels of wild type TP53 protein and mRNA were lower in ESCC tissues compared to the normal esophageal epithelium. In addition, the mRNA and protein expression levels were reported as downregulated further in tumors with metastasis than in tumors without. In conclusion, miR-612 is identified as associated with ESCC development and metastasis, likely through the regulation of TP53 expression, which could be a potential therapeutic target.
\end{abstract}

\section{Introduction}

Esophageal cancer is the eighth most common cancer and the sixth most common cause of cancer deaths worldwide (1). A total of $70 \%$ of all esophageal cancer worldwide occurs in China, of which $90 \%$ are esophageal squamous cell carcinoma (ESCC). The main treatment of the cancer has been the combination of surgery, radiation and chemotherapy, yet the 5 year

Correspondence to: Dr Qing Sun, Department of Pathology, Qianfoshan Hospital Affiliated with Shandong University, 16766 Jingshi Road, Jinan, Shandong 250014, P.R. China

E-mail: qingsw99@163.com

Key words: esophageal squamous cell carcinoma, microRNA-612, TP53, lymph node metastasis survival rate is $<15 \%$ (2). Invasion and metastasis is the main contributor to the outcome (3).

miRNAs are small non-coding RNAs that regulate mRNA levels at the post-transcriptional level by either degrading mRNA or blocking its translation, thus affecting protein production. Growing evidence suggests that miRNAs serve a key role in the expression regulation of multiple genes and related signal transduction pathway synchronization, as well as in various diseases, including cancer development and progression (4,5). Using Ambion bioarrays, Feber et al (6) compared the expression patterns of 328 miRNAs among adenocarcinoma, ESCC, Barrett esophagus, high-grade dysplasia and normal epithelium samples, and demonstrated miR-203 and miR-205 were downregulated and miR-21 was upregulated in both ESCC and adenocarcinoma sample compared to the normal epithelium. Furthermore, Guo et al (7) identified potentially important miRNAs in ESCC. The expression of three miRNAs were upregulated, including hsa-miR-25, hsa-miR-424 and hsa-miR-151, and four were downregulated, including hsa-miR-100, hsa-miR-99a, hsa-miR-29c and mmu-miR-140. These miRNAs may be used as diagnostic biomarkers to separate ESCC from normal epithelium. In addition, elevated expression of miR-103/107 was strongly correlated with poor survival in patients with ESCC from mono-variant and multi-variant analysis. Increased expression of miR-129 was reported as associated with poor survival of surgically treated ESCC patients (8).

In spite of these advances, consistent findings across studies are lacking. Most studies do not have functional or mechanistic investigation for the potential markers identified; association with overall survival can be challenging as it can be confounded by many other factors. In the present study, miRNAs in ESCC tissues were compared with lymph node metastasis and those without, and associated with tumor metastasis by whole genome miRNA microarray analysis. miR-612 was indicated as highly expressed in tumors with metastasis. In addition, the authors investigated the mechanisms of miR-612 in tumor invasion and metastasis in both cell lines and tumor tissues. The result demonstrated that miR-612 is positively correlated with tumor aggressiveness, which was aborted by an miR-612 inhibitor. The expression of miR-612 is inversely correlated with P53 mRNA and protein expression. 


\section{Materials and methods}

Selection of miRNAs associated with tumor metastasis. The research was approved by the Ethics Committee of Qianfoshan Hospital (Jinan, China), and all clinical investigation were conducted according to the principles expressed in the Declaration of Helsinki. All participants in the study had signed written informed consents.

To identify the candidate miRNAs that are associated with tumor metastasis, the authors first conducted miRNA profiling for tumors with lymph node metastasis and for those without. The fresh frozen tissues of 10 primary ESCC, four with lymph node metastasis and six without, were selected from the tumor tissue collection bank in the Department of Pathology, Qianfoshan Hospital (Jinan, China). The tissue samples were obtained from radical esophagectomy performed in the same hospital, and patient information was retrieved from medical records and pathological reports. No other treatments were given prior to surgery. Tumor tissue $(\sim 100 \mathrm{mg})$ was taken from the region without hemorrhage and necrosis. The normal esophageal epithelium was taken from $\sim 3-5 \mathrm{~cm}$ away from the edge of the tumor. The samples were fast frozen in nitrogen within 15 min following being resected and stored at $-80^{\circ} \mathrm{C}$ for later use.

RNA extraction. Tissue $(30 \mu \mathrm{g})$ was taken from tumor and normal tissues for each case and total RNA was isolated using TRIzol (Invitrogen; Thermo Fisher Scientific, Inc., Waltham, MA, USA) and miRNeasy mini kit (Qiagen GmbH, Hilden, Germany) according to manufacturer's instructions, which efficiently recovered all RNA species, including miRNAs. RNA quality and quantity was measured by using a Nanodrop spectrophotometer (ND-1000, Nanodrop; Thermo Fisher Scientific, Inc., Wilmington, DE, USA), and RNA integrity was determined by agarose gel electrophoresis. The $\mathrm{OD}_{260} / \mathrm{OD}_{280}$ ratios ranged from 1.8 to 2.01 and no DNA contamination and RNA degradation were observed.

RNA labeling. miRCURY TM isolation (Exiqon A/S, Vedbaek, Denmark) was used. Of each sample, $1 \mu \mathrm{g}$ was 3'-end-labeled with Hy3TM fluorescent label (Exiqon A/S), using $\mathrm{T}_{4}$ RNA ligase by the following procedure: RNA in $2.0 \mu \mathrm{l}$ water was combined with 1.0 $\mu \mathrm{l}$ CIP buffer and CIP (Exiqon A/S). The mixture was incubated for $30 \mathrm{~min}$ at $37^{\circ} \mathrm{C}$, using $\mathrm{T}_{4}$ RNA ligase, $1.5 \mu$ l fluorescent label (Hy3TM), $2.0 \mu 1$ DMSO (Beijing Solarbio Science \& Technology Co., Ltd., Beijing, China), $2.0 \mu 1$ labeling enzyme were added into the mixture. The labeling reaction was incubated for $1 \mathrm{~h}$ at $16^{\circ} \mathrm{C}$, and terminated by incubation for $15 \mathrm{~min}$ at $65^{\circ} \mathrm{C}$.

miRNA microarray. The fifth generation of miRCURY ${ }^{\mathrm{TM}}$ LNA Array (version, 14.0; Exiqon A/S) was used, which contains $>1,891$ capture probes, covering all human, mouse and rat microRNAs annotated in miRBase 14.0 (http://www.mirbase.org/), as well as all viral microRNAs related to these species. Following the completion of the labeling procedure, the Hy3TM-labeled samples were hybridized on the miRCURY ${ }^{\text {Tм }}$ LNA Array, according to manufacturer's instructions. The total $25 \mu \mathrm{l}$ mixture from Hy3TM-labeled samples with $25 \mu$ l hybridization buffer were first denatured for $2 \mathrm{~min}$ at $95^{\circ} \mathrm{C}$, then placed on ice for $2 \mathrm{~min}$ and then hybridized to the microarray for 16-20 h at $56^{\circ} \mathrm{C}$ in a 12-Bay Hybridization Systems (NimbleGen; Roche Diagnostics, Basel, Switzerland), which provides an active mixing action and constant incubation temperature to improve hybridization uniformity and enhance the signal. Following hybridization, the slides were washed several times using Washing buffer kit (Exiqon A/S), and finally dried by centrifugation for $5 \mathrm{~min}$ at $95 \mathrm{x} \mathrm{g}$. Then the slides were scanned using the Axon GenePix 4000B microarray scanner (Molecular Devices, LLC, Sunnyvale, CA, USA).

miRNA array data analysis. Scanned images were then imported into GenePix Pro 6.0 software (Molecular Devices, LLC) for grid alignment and data extraction. Replicated miRNAs were averaged and miRNAs with intensities $>50$ arbitrary units in all samples were chosen for calculating normalization factor. Expressed data were normalized using the Median normalization. Hierarchical clustering was performed using TIGR MeV software (version, 4.6; J. Craig Venter Institute, Rockville, MD, USA). Differentially expressed miRNAs between normal and tumor tissues were identified by combination of volcano plot filtering and PAM (Prediction Analysis of Microarrays; version 2.1; http://statweb.stanford. edu/ tibs/PAM/) (9). The selected genes were illustrated in heat map by MEV software. Genes with differential a $\mathrm{P}<0.05$ and fold change $>2$ were selected for further investigation.

Reverse transcription-quantitative polymerase chain reaction (RT-qPCR) validation. To validate miR-612 by RT-qPCR, the authors chose additional 70 samples, including 26 cases of ESCC with lymph node metastasis, 24 cases without, and 20 cases of normal esophageal epithelial tissues. Together with the 20 samples, which had been analyzed by miRNA microarray, a total of 90 samples were used for RT-qPCR validation. U6 was used as reference for normalization (10). Directional primers were designed according to the known miRNA sequences: hsa-miR-612 forward, 5'GCTGGGCAGGGCTTCT3' and reverse, 5'CAGTGCGTGTCGTGGAGT3'; U6 forward, 5'GCTTCGGCAGCACATATACTAAAAT3' and reverse, 5'CGCTTCACGAATTTGCGTGTCAT3'. RNA was reverse transcribed to cDNA and then measured by RT-qPCR at $90^{\circ} \mathrm{C}$, denatured for $10 \mathrm{sec}, 60^{\circ} \mathrm{C}$ for $20 \mathrm{sec}, 72^{\circ} \mathrm{C}$ for $20 \mathrm{sec}$ and $78^{\circ} \mathrm{C}$ for $20 \mathrm{sec}$ for 45 cycles to establish miR-612 and U6 standard and melting curves for each sample.

\section{RT-qPCR examining TP53 mRNA expression in EC109 cells} transfected with miR-612. EC109 cells were transferred into a $25 \mathrm{ml}$ flask the day prior to transfection when cells were $\sim 80 \%$ confluent. These cells were transfected with $2 \mu \mathrm{g}$ pSilencer/miR-612 (to express miR-612) and $2 \mu \mathrm{g}$ control plasmid pSilencer/NC, respectively. At $24 \mathrm{~h}$, RNAs were extracted from the EC109 cells by TRIzol reagent and reverse transcribed to cDNA as before, to detect the TP53 expression levels in the EC109 cells.

mRNA target prediction. The authors applied TargetScan (http://www.targetscan.org/), Pictar (http://www.pictar.org/), miRanda (http://www.microrna.org/microrna/home.do) and MirTarget 2 (http://mirdb.org/miRDB/download.html) 
analysis tools to predict the miRNA targets and selected the more reliable targets that were predicted by at least three out of the four tools.

Cell lines. Human esophageal carcinoma cell lines EC109 and EC9706 were purchased from the Type Culture Collection of the Chinese Academy of Sciences (Shanghai, China), which were cultured in Dulbecco's modified Eagle's medium (DMEM) with $10 \%$ fetal bovine serum at $37^{\circ} \mathrm{C}$ and $5 \% \mathrm{CO}_{2}$. All experiments were carried out when the cells reached $60 \%$ confluency.

Main reagents. miR-612 inhibitor (anti-sense) and its negative control, TRIzol reagent, Lipofectamine 2000, and plasmids either with miR-612 (pSilencer/miR-612) or with negative control (pSilencer/NC) were purchased from Shanghai GenePharma Co., Ltd (Shanghai, China). The MicroRNA extracting kit (miRNeasy Mini Kit) was from Qiagen. The TaqMan ${ }^{\circledR}$ MicroRNA Reverse Transcription kit and Real-time PCR reagent (including miR-612 Assay, U6 Assay and TaqMan Universal Master Mix II, no UNG) were from Thermo Fisher Scientific, Inc. The miR-612 validation primer and the internal reference U6 were bought from Guangzhou Funeng Gene Company (Guangzhou, China). Transwell (Millicell Hanging Cell Culture Inserts) and ECM matrix (ECM Cell Attachment Matrix) were from EMD Millipore (Billerica, MA, USA). Fetal bovine serum (FBS) was obtained from Tianjin Haoyang Biological Products Technology Co., Ltd. (Tianjin, China). DMEM was purchased from Gibco; Thermo Fisher Scientific, Inc. The primary antibody against TP53 was a mouse anti-human monoclonal antibody, the internal reference was a rabbit anti-human GAPDH monoclonal antibody (Beijing Biosynthesis Biotechnology Co., Ltd., Beijing, China), and the secondary antibodies were rabbit anti-mouse $\mathrm{IgG}$ and sheep anti-rabbit IgG (Beijing Zhongshan Jinqiao Biotechnology Co., Ltd., Beijing, China) The luciferase reporter assay kit was from Invitrogen; Thermo Fisher Scientific, Inc.

Scratch test to measure migration ability of cells. EC109 cells were divided into 3 groups, with three replicates per group: miR-612 inhibitor group, negative control group and blank group. At $24 \mathrm{~h}$ following transfection and incubation at $37^{\circ} \mathrm{C}$ with $5 \% \mathrm{CO}_{2}$ to form a single layer of cells, a scratch line was made at the bottom of each well with a $10 \mu$ l pipette tip. The cells were washed gently to remove the deciduous cells and culture medium was replaced with serum-free medium. Migration vs. scratch distance was observed and measured at 0,36 and $72 \mathrm{~h}$, respectively.

Tumor cell migration test. EC109 cells were separated into 3 groups, three replicates each: miR-612 inhibitor group, negative control group and blank group. After being transfected for $24 \mathrm{~h}$, the EC109 cells were digested with trypsin and suspended with serum-free DMEM. The cell suspension, $\left(2 \times 10^{4}\right.$ cells $/ \mathrm{ml} /$ well in $\left.200 \mu \mathrm{l}\right)$, was added to the upper chamber of the Transwell and $900 \mu 1$ DMEM with 5\% FBS was added to lower chamber. After having been incubated for $6 \mathrm{~h}$ at $37^{\circ} \mathrm{C}$, the remaining cells in the upper chamber were wiped off with a wet cotton ball. The cells on the wet cotton ball were collected on a glass slide, fixed with carbinol for
20 min, stained with $0.1 \%$ crystal violet for $20 \mathrm{~min}$., and then washed with PBS 3 times. The number of cells that migrated to the lower chamber was counted at x 200 magnification using an inverted microscope. A total of 10 random fields of view for each sample were used for counting.

Tumor cell invasion test. The tips and Eppendorf tubes were pre-cooled at $4^{\circ} \mathrm{C}$ overnight. Matrigel $(1,000 \mu \mathrm{g} / \mathrm{ml})$ was diluted to $0.2 \mu \mathrm{g} / \mu 1$ with DMEM operated on ice. A total of $50 \mu \mathrm{l}$ Matrigel was added to the upper chamber of the Transwell and air dried at $4^{\circ} \mathrm{C}$ overnight. The excess DMEM was removed and then hydrated with DMEM at $37^{\circ} \mathrm{C}$ for $1 \mathrm{~h}$. EC109 cells were divided into 3 groups, three replicated each: The miR-612 inhibitor group, negative control group and blank group. After being incubated for $24 \mathrm{~h}$ at $37^{\circ} \mathrm{C}$, the remaining cells in the upper chamber were wiped off with a wet cotton ball. The number of cells which migrated to the lower chamber was counted at x200 magnification in an inverted microscope. A total of 10 views for one sample were used for counting.

Enhanced green fluorescent protein (EGFP) fluorescent reporter carrier assay. This experiment was conducted to demonstrate that TP53 is the direct target of miR-612. EC109 cells were plated on a 48 -well plate $\left(1.5 \times 10^{4}\right.$ cells/well $)$ one day before transfection, with the cell concentration at about $80 \%$ confluency, and then co-transfected with Lipofectamine 2000 and pSilencer/miR-612 or pSilencer/NC. Additionally, TP53 3'UTR mutant (pcDNA3-Egfp-P53-3'UTR mut) and wild-type (pcDNA3-Egfp-P53-3'UTR; $0.3 \mu \mathrm{g}$ ) plasmids were also included to test whether the mutation at the binding site affects miR-612 binding and subsequent TP53 activity. There were nine parallel wells in each group. Serum was added to the wells $4 \mathrm{~h}$ after transfection to a final serum concentration of $10 \%$. At $48 \mathrm{~h}$ following transfection, cells were treated with radioimmunoprecipitation assay buffer $(150 \mathrm{mmol} / \mathrm{l}$ $\mathrm{NaCI}, 50 \mathrm{mmol} / \mathrm{l}$ Tris-HCl, pH 7.2,1\% Triton X-100 and $0.1 \%$ SDS), centrifuged to separate the supernatant at 9,503 $\mathrm{x} g$ for $10 \mathrm{~min}$. The expression levels of green fluorescent proteins were measured with fluorescence spectrophotometer F-4500 at $484 \mathrm{~nm}$ for excitation and $510 \mathrm{~nm}$ for emission.

Western blotting. Western blotting was conducted to measure TP53 protein expression in tumor and normal tissues. The same 90 samples that had been used for RT-qPCR validation were used for the measurement of TP53 protein expression. Fresh frozen tissue (1-2 g) of each sample was added to the precooled buffer solution. Ultrasonic dispersion was performed in an ice-water bath and then centrifuged at $13,684 \mathrm{x}$ g at $4^{\circ} \mathrm{C}$. Protein $(\sim 100 \mu \mathrm{g})$ was separated by electrophoresis in $12 \%$ SDS-PAGE gel, then transferred to a polyvinylidene difluoride (PVDF) membrane, blocked with $5 \%$ skim milk at $4^{\circ} \mathrm{C}$ overnight. The primary antibodies [mouse anti-human wild type TP53 monoclonal antibody (cat. no. D199442; 1:50,000; Beijing Biosynthesis Biotechnology Co., Ltd.) and rabbit anti-human GAPDH monoclonal antibody (cat. no. D110016; 1:2,000; Beijing Biosynthesis Biotechnology Co., Ltd.)] were added, incubated for $2 \mathrm{~h}$ at $37^{\circ} \mathrm{C}$. The PVDF membrane was washed with $0.1 \%$ TBS-Tween-20 three times, each for $10 \mathrm{~min}$ at room temperature. The secondary antibodies [rabbit anti-mouse IgG (cat. no. ZDR-5109; horseradish peroxidase-conjugated; 
1:300,000; Beijing Zhongshan Jinqiao Biotechnology Co. Ltd.) sheep anti-rabbit IgG (cat. no. ZB-5118; 1:10,000; Beijing Zhongshan Jinqiao Biotechnology Co. Ltd.)] were added, and incubated for $2 \mathrm{~h}$ at $37^{\circ} \mathrm{C}$. The membrane was washed with TBS-Tween 20 and Immobilon Western Chemiluminescent HRP substrate (EMD Millipore) was added. Clear protein bands on transfected membrane indicated positive results. A ChemiDoc XRS+ image analyzer (Bio-Rad Laboratories, Inc., Hercules, CA, USA) and Image Lab 4 software (Bio-Rad Laboratories, Inc.) was used to measure the gray level of the samples.

Western blotting identification of TP53 protein expression in EC109 cells transfected with miR-612. EC109 cells were lysed $48 \mathrm{~h}$ after the transfection with miR-612, centrifuged for supernatant at $13,684 \mathrm{x}$ g for $10 \mathrm{~min}$, electrophoresed on a $10 \%$ SDS-PAGE gel, transferred to nitrocellulose membrane, blocked with $5 \%$ skim milk at $4^{\circ} \mathrm{C}$ overnight. The primary antibodies and the secondary antibodies were added, respectively, as described earlier.

Statistical analysis. Measurement data are expressed as the mean \pm standard deviation. Comparisons among three groups were performed by one-way analysis of variance (ANOVA) followed by Fisher's least significant difference and Student-Newman-Keuls post hoc tests. Comparisons between two groups were made with unpaired Student's t-test. In all cases, $\mathrm{P}<0.05$ was considered to indicate a statistically significant difference.

\section{Results}

miR-612 is highly expressed in metastatic tumor tissues. miRNA expression profiling was investigated for $10 \mathrm{ESCC}$ samples, five with metastasis and five without, using an miRCURY LNA Array. Differential expression analysis indicates that 16 miRNAs are significantly different between the two groups, $\mathrm{P}<0.05$ and fold change $(\mathrm{FC})>2$, among which 13 were upregulated and three were downregulated in the tumors with metastasis. The authors further conducted non-parametric PAM analysis, and miR-612 remained a strong predictor for metastasis phenotype (Fig. 1).

Validation of miR-612 by RT-qPCR. RT-qPCR was performed to validate the expression of miR-612 in 70 samples. The $\mathrm{Cq}$ value was first obtained by comparing the amplification curves with threshold value. The FC between miR-612 and U6 was obtained by the $2^{-\Delta \Delta C \mathrm{Cq}}$ method where a value $>2$ indicates upregulation and $<0.5$ for downregulation. Consistent with microarray results, miR-612 increased $>$ five-folds in tumors with lymph node metastasis (LymphoMet), when compared with the tumors without $(\mathrm{P}<0.05$; Fig. 2).

miR-612 inhibitor transfection into EC109 and EC9706 cell lines. The expression of miR-612 in the normal esophageal epithelium and cancer cell lines EC109 and EC9706 was measured using RT-qPCR. Compared to the normal esophageal epithelium cell line (established in the authors' laboratory), both EC109 and EC9706 cell lines presented increased expression of miR-612, 4.49- and 3.05-fold higher, respectively ( $\mathrm{P}<0.05$; Fig. 3A). EC109 was then selected for the transfection experiment with three conditions groups created: miR-612 inhibitor (150 nmol/l), negative control with scrambled miRNA (NC), and a blank control (culture media only). After $72 \mathrm{~h}$ of the treatments, RT-qPCR was used to measure the miR-612 levels. Compared to those prior to the treatment, the group with the miR-612 inhibitor had significantly reduced expression of miR-612 $(\mathrm{P}<0.05)$, while the two control groups saw no change $(\mathrm{P}>0.05$; Fig. $3 \mathrm{~B})$.

Reduced healing in the miR-612 suppressed group as a result of scratch healing. After scratching, the experimental group whose miR-612 was inhibited by siRNA had a significantly reduced healing rate than in the negative control or blank control group $(\mathrm{P}<0.05$ and $\mathrm{P}<0.01$, respectively; Fig. 4). Healing rate was measured by the width of the scratch, which was defined as $0 \%$ at $0 \mathrm{~h}$, and $100 \%$ when the cells were kept confluent.

Cancer cells infected with miR-612 inhibitor had reduced invasive and migratory abilities. The invasive and migratory abilities for EC109 cells were measured through the Transwell experiment following transfecting the cells with the miR-612 inhibitor along with scrambled siRNA as negative control and blank control. The cells transfected with the miR-612 inhibitor had significantly reduced invasive ability, as demonstrated by the fewer number of cells migrated out of wells, when compared with the cells without specific inhibition, either by scrambled miRNA or blank media (both $\mathrm{P}<0.01$; Fig. 5).

Prediction and evaluation of target genes of miR-612. TargetScans, Pictar and miRanda and MirTarget 2 were used to search for the mRNA targets of miR-612 and the targets that were predicted by all the prediction tools were evaluated (45 predicted miRNA target genes). With consideration of the literature reports for esophageal squamous cell carcinoma-associated genes (11-17), it was identified that TP53 is likely the most important target of miR-612 due to its significant role in cancer development. The current research was focused on the relationship of TP53 and miR-612.

TP53 is targeted by miR-612 as demonstrated by the EGFP fluorescent reporter carrier experiment. The miR-612 expressionplasmidpSiIencer/miR-612 was mixed with the fluorescent reporter carrier plasmid pcDNA3-Egfp-P53-3'UTR or pcDNA3-Egfp-P53-3'UTRmut and transfected into EC109 cells. The pSilencer/NC plasmid with fluorescent reporter carrier plasmid pcDNA3-Egfp-P53-3'UTR or pcDNA3-Egfp-P53-3'UTRmut was used as the control. After $48 \mathrm{~h}$ transfection, green fluorescent protein expression levels were measured by a fluorescence spectrophotometer. The cells infected with miR-612 expression plasmid had reduced TP53 3'UTR reporter plasmid GFP by $29.3 \%$ $(\mathrm{P}<0.05)$, compared to the control group, suggesting that the expressed miR-612 acted on the $3^{\prime}$ untranslated region of TP53 transcripts and caused reduced expression of GFP. However, the pSilencer/miR-612 or control plasmid pSilencer/NC transfected with mutant TP53 (3'UTR pcDNA3-Esfp-P53-3'UTRmut) had no inhibitory effect on miR-612 ( $\mathrm{P}>0.05$; Fig. 6A and B). 


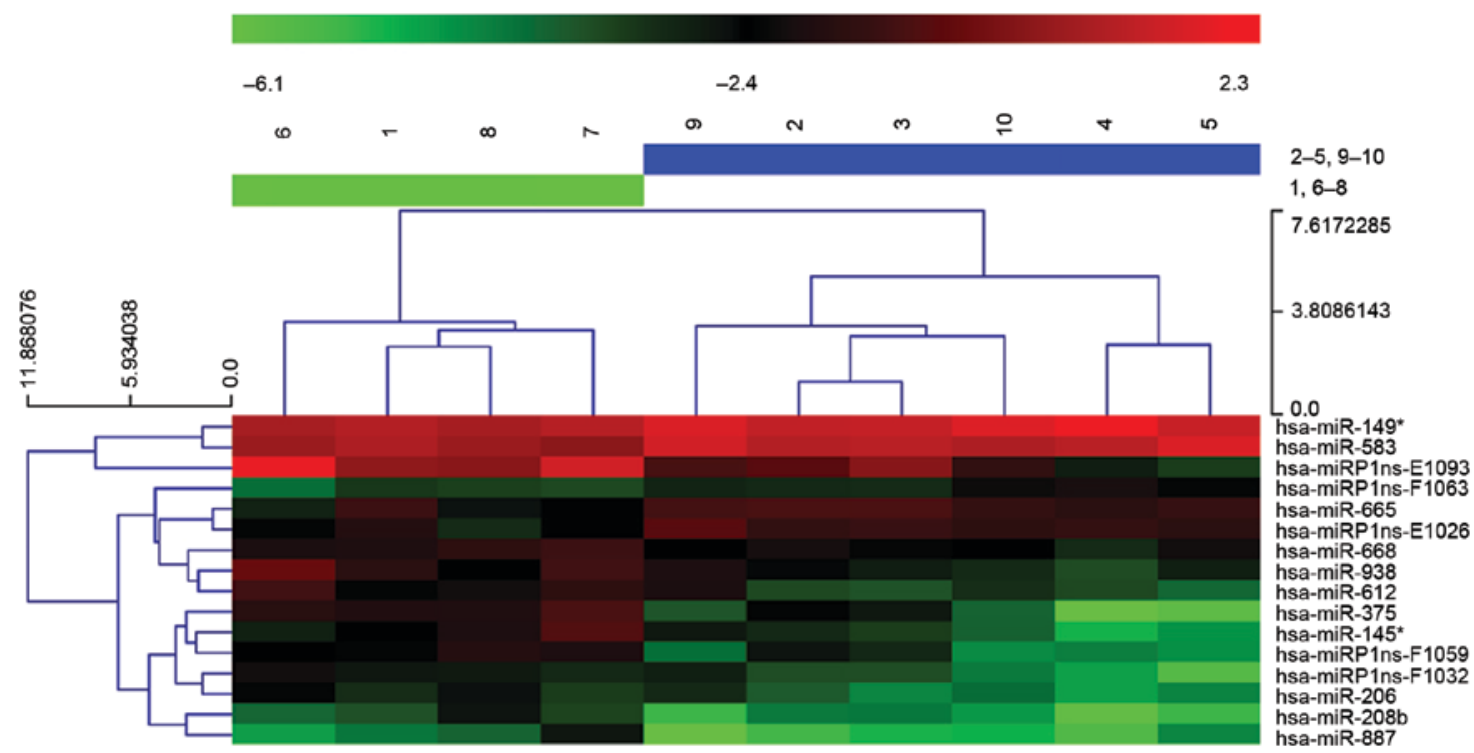

Figure 1. Heatmap of differentially expressed miRs between metastatic and non-metastatic esophageal carcinoma. Sample numbers at the top: 1 and 6-8, non-metastatic group; 2-5 and 9-10, metatatic group. miR, microRNA.

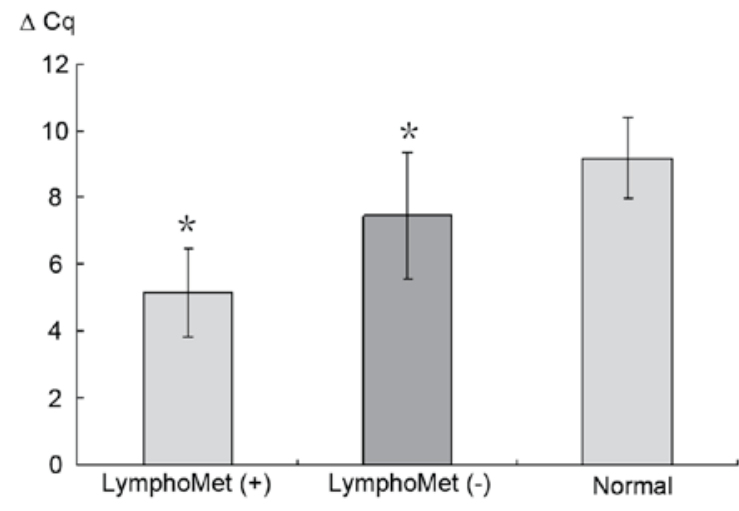

Figure 2. Reverse transcription-quantitative polymerase chain reaction analysis was used to validate the expression of miR-612 in the 70 samples. U6 was used as a control. The expression of miR-612 increased more than five-folds in tumors with LymphoMet compared to the tumors without. Data are presented as means \pm standard deviation. ${ }^{*} \mathrm{P}<0.05$ vs. normal group. miR, microRNA; LymphoMet, lymph node metastasis.

Reduced wild type P53 protein expression in tumors and metastatic tumors of esophageal squamous cell carcinoma by western blotting. A western blot analysis was conducted to measure the wild type P53 protein expression in: 30 cases of ESCC with lymph node metastasis, 30 cases without, and 10 cases of normal esophageal epithelial tissues. The average grey value was obtained for each case and one-way ANOVA, with post hoc Fisher's least significant difference and Student-Newman-Keuls tests, was performed for differential protein levels among the three groups. Both the tumors with and without metastasis presented significantly reduced TP53 protein expression compared to the normal tissues (75.5\%, 46.7\% reduction, respectively, $\mathrm{P}<0.05$, Fig. $7 \mathrm{~A}$ and $\mathrm{B}$ ). The tumors with metastasis had further reduced wide type TP53 expression compared to the tumors without metastasis.

miR-612 downregulates wild type P53 mRNA expression in EC109 cells. When the pSilencer/miR-612 plasmid and the
A $\Delta \mathrm{Cq}$

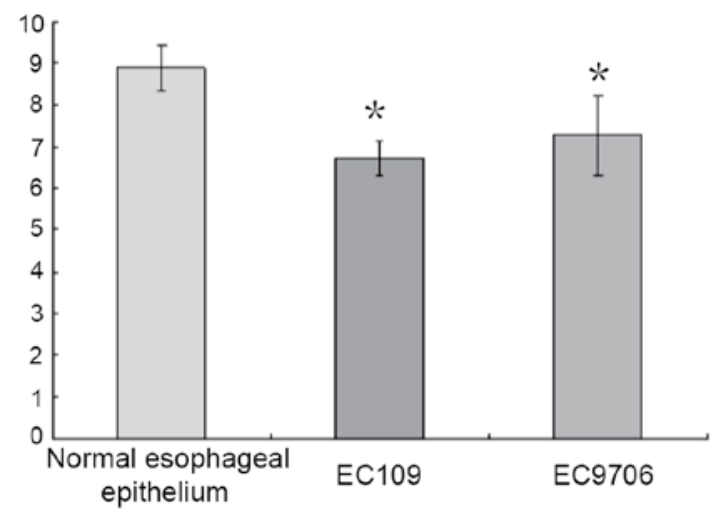

B $\quad \triangle \mathrm{Cq}$

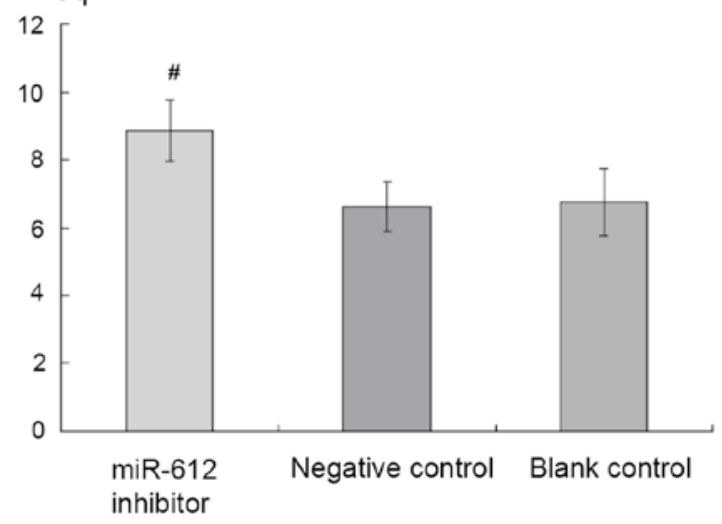

Figure 3. Reverse transcription-quantitative polymerase chain reaction analysis was used to validate the expression of miR-612 in (A) normal esophageal epithelium, cancer cell line EC109 and EC9706 cells and in (B) synthesized miR-612 inhibitor, negative control and blank control. EC109 and EC9706 had increased expression of miR-612 when compared to the normal esophageal epithelium (4.49- and 3.05-fold higher). The group with the miR-612 inhibitor had significantly reduced expression of miR-612, while the two control groups essentially had no change. Data are presented as means \pm standard deviation ${ }^{*} \mathrm{P}<0.05$ vs. normal group; ${ }^{~} \mathrm{P}<0.05$ vs. negative and blank control groups. miR, microRNA. 

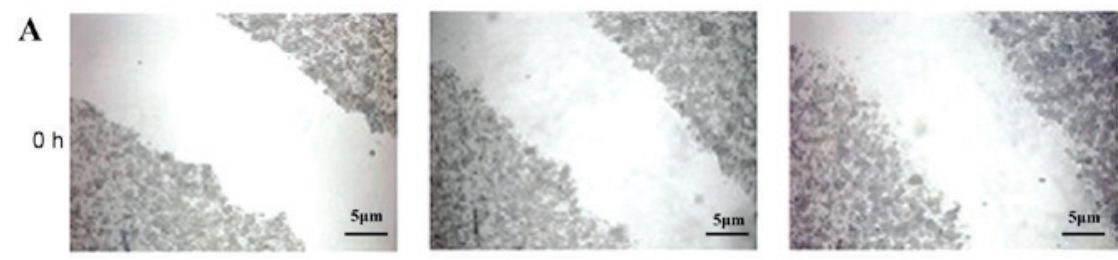

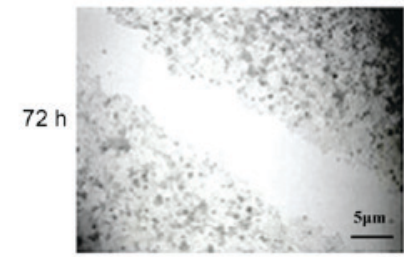

Transfection group

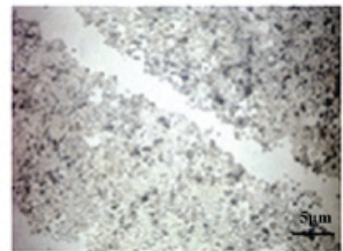

Negative control group

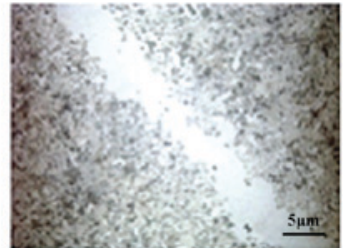

Blank control group

B

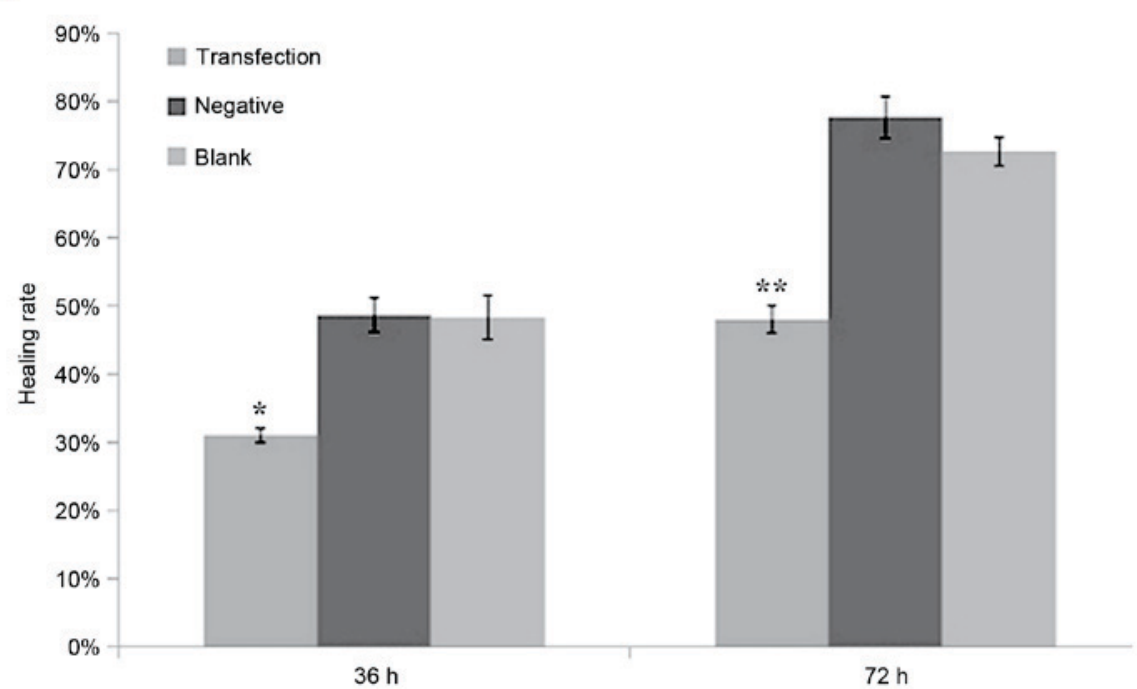

Figure 4. (A) Scratch test of the transfection group, negative control group and blank control group at 0 and $72 \mathrm{~h}$. The transfection group had a significantly reduced healing rate than in the negative control or blank control group $72 \mathrm{~h}$ after scratch. (B) Scratching healing rates at 36 and $72 \mathrm{~h}$ among three experimental groups. The transfection group has significant lower healing rate than the negative control or blank control group. Data are presented as means \pm standard deviation. ${ }^{*} \mathrm{P}<0.05$ and ${ }^{* *} \mathrm{P}<0.01$ vs. negative and blank control groups.

negative control pSilencer/NC plasmid were used to transfect EC109 cells, TP53 mRNA levels, measured by RT-qPCR, were significantly reduced in the pSilencer/miR-612 infected cells compared to the negative controls (2.2-fold less; $\mathrm{P}<0.001$; Fig. 8A).

miR-612 downregulates wild type P53 protein expression in EC109 cells. The authors further measured the protein expression of TP53 by western blotting. At $48 \mathrm{~h}$ following transfection with the pSilencer/miR-612 plasmid and negative control pSilencer/NC plasmid of EC109 cells, respectively, TP53 expression was measured through western blotting and showed that comparing to the control group, the TP53 expression in pSilencer/miR-612 group was significantly reduced $(\mathrm{P}<0.01$, Fig. 8B and $\mathrm{C})$.

\section{Discussion}

A previous study demonstrated that $>50 \%$ of miRNAs are located in tumor-associated gene loci or fragile sites in the genome (18). miRNAs can act as a tumor suppressor that inhibits the activities of oncogenes or they can involve in cell cycle, regulate apoptosis, angiogenesis and tumor metastasis $(4,5)$. However, our understanding for these functions is limited to a very few miRNAs and more research on more miRNAs is needed.

There has been growing interest in the role of miRNAs in ESCC development for the past few years. The first genome wide survey using miRNA microarray demonstrated that 46 miRNAs are differentially expressed compared to its adjacent normal epithelium (7). The study by Feber et al (6) demonstrates that miR-194, miR-192 and miR-200c express much higher in adenocarcinoma than in squamous cell carcinoma while miR-21, miR-205, miR-203 and miR-93 are dramatically different between tumor and normal tissue. The expression pattern changes could be potentially used to differentiate tumor from normal tissue for esophageal cancer diagnosis (19). For example, ANXA1 is a tumor suppressor gene that has a role in inhibiting cell proliferation and promoting apoptosis. miR-196, a miRNA that can target ANXA1, may inhibit the expression of ANXA1 and cause the development of esophageal carcinoma (19). LATS2 is another tumor suppressor and it targeting 


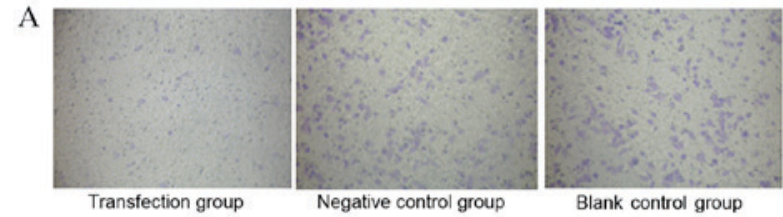

B

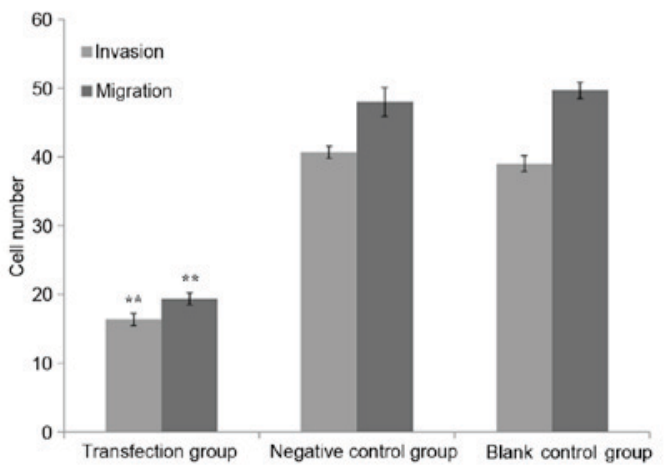

Figure 5. Tumor cells invasive and metastatic ability measurement. (A) Matrigel invasion assay demonstrated that EC109 cells transfected with miR-612 inhibitors exhibited reduced invasive ability. (B) Quantified migration and invasion results. The tumor cells transfected by miR-612 inhibitors have lower migration and invasion capabilities than the negative control or blank control group. Data are presented as means \pm standard deviation. ${ }^{* *} \mathrm{P}<0.01$ vs. negative and blank control groups.
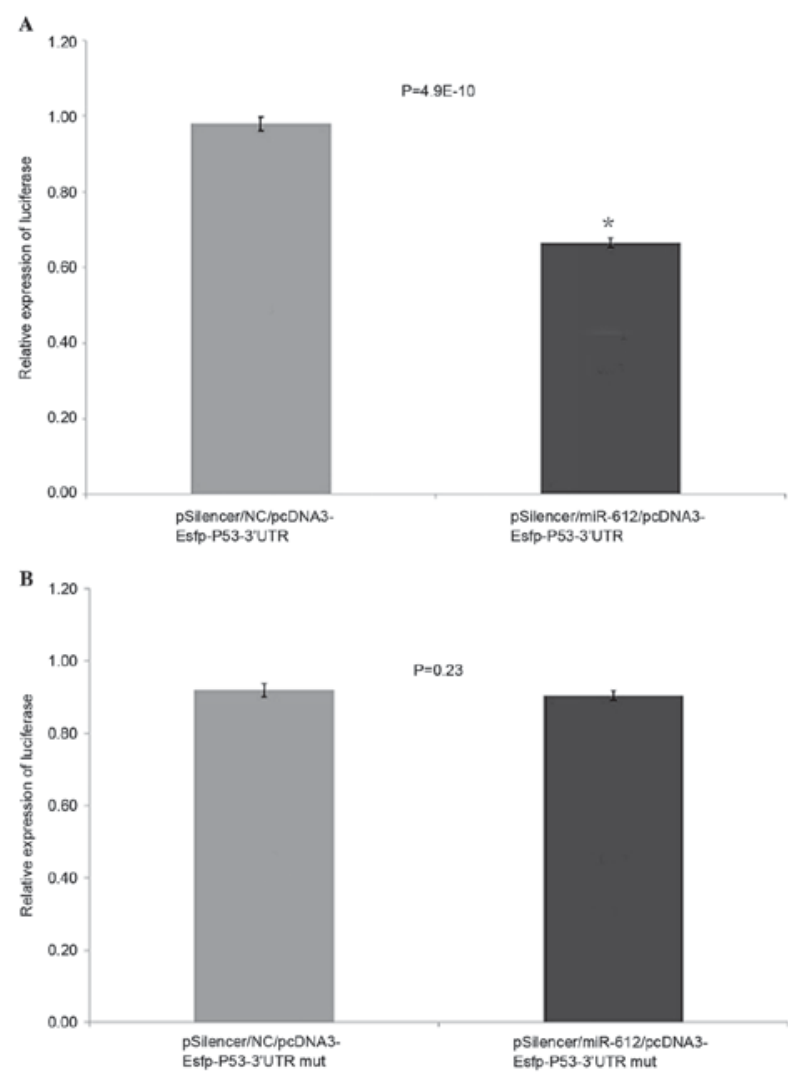

Figure 6. EGFP fluorescent reporter carrier experiment. (A) miR-612 had reduced TP53 3'UTR reporter plasmid green fluorescent protein than blank control group (pSilencer/NC/pcDNA3-Esfp-P53-3'UTR). (B) miR-612 did not reduce TP53 3'UTR mut reporter plasmid green fluorescent protein. Data are presented as means \pm standard deviation. ${ }^{*} \mathrm{P}<0.05$ vs. pSilencer/NC/pcDNA3-Esfp-P53-3'UTR. EGFP, enhanced green fluorescent protein; miR, microRNA; UTR, untranslated region; NC, negative control.
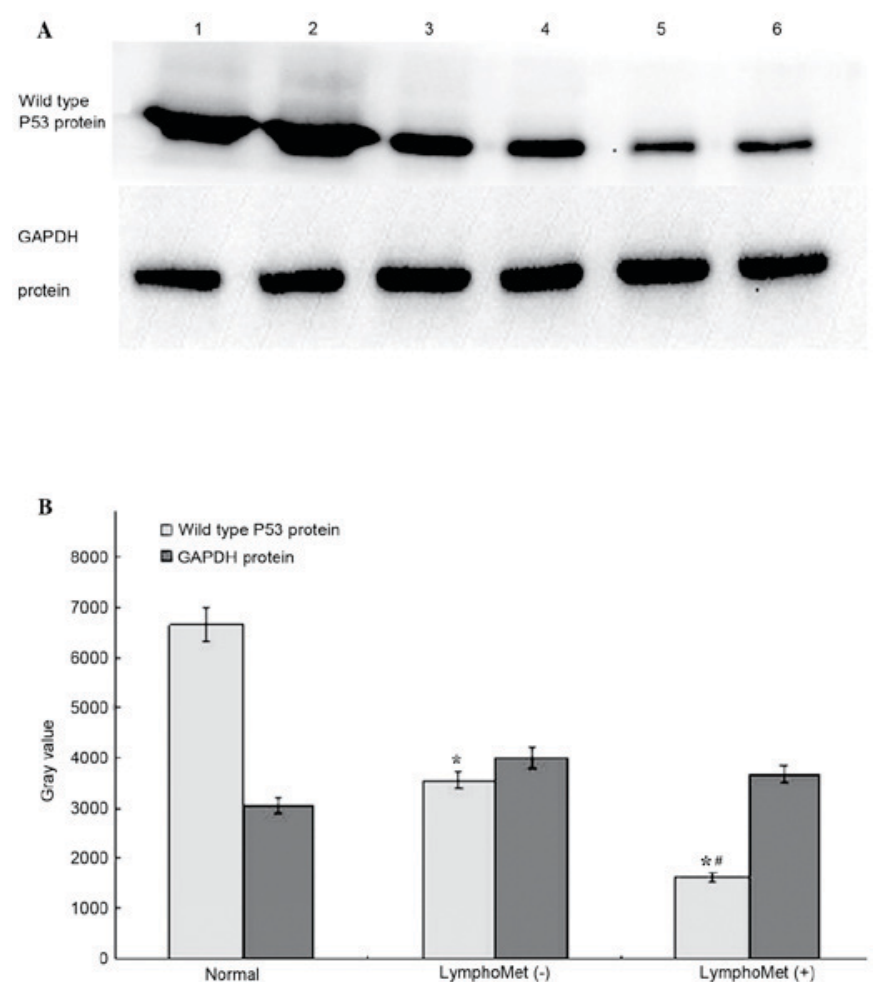

Figure 7. (A) Western blot analysis to measure the TP53 protein expression in 30 cases of ESCC with lymph node metastasis, 30 cases without and 10 cases of normal esophageal epithelial tissues. (B) Both the tumors with metastasis and the tumors without metastasis had significantly reduced TP53 protein expression compared to the normal tissues $(\sim 75.5 \%, 46.7 \%$ reduction, respectively). The tumors with metastasis had further reduced TP53 expression compared to the tumors without metastasis ( $\sim 54.1 \%$ reduction). Data are presented as means \pm standard deviation. ${ }^{~} \mathrm{P}<0.05$ vs. normal group; ${ }^{~} \mathrm{P}<0.05$ vs. LymphoMet (-) group.

miR-373 may have a role in SCC development through downregulating this gene (20) The study by Hiyoshi (21) indicates that miR-21 is not only overexpressed in primary tumors, but also in metastatic tumors. miR-21 mediates ESCC development and progression through suppressing the expression of PDCD4. In the study of SSC sub-clone, Tian et al (22) find that miR-10b serves a role in tumor cell invasion and metastasis through the inhibition of tumor suppression gene KLF4. In spite of these progresses, prominent and specific miRNAs for SSC are still lacking $(23,24)$.

In the present study, miRNA expression was profiled between ESCC with and without lymph node metastasis and identified 63 differentially expressed miRNAs. Further analyses through PAM and target prediction revealed miR-612 as a potential regulator in ESCC development and metastasis. The authors' functional validation further indicated that miR-612 as a tumor promoter was likely mediated through downregulating TP53. To the best of the authors' knowledge, the present study is the first to establish the link of miR-612 with ESCC metastasis.

The function of miR-612 and downstream regulation pathway is largely unknown. This microRNA coding sequence is located at chromosome 11q13.1, with mature sequence gcugggcagggcuucugagcuccu. Tian et al (25) reported that the nucleotide sequence of miR-612 is the same as miRNA-1285 but it does not bind to the P53 3'UTR. Tao et al (26) report 


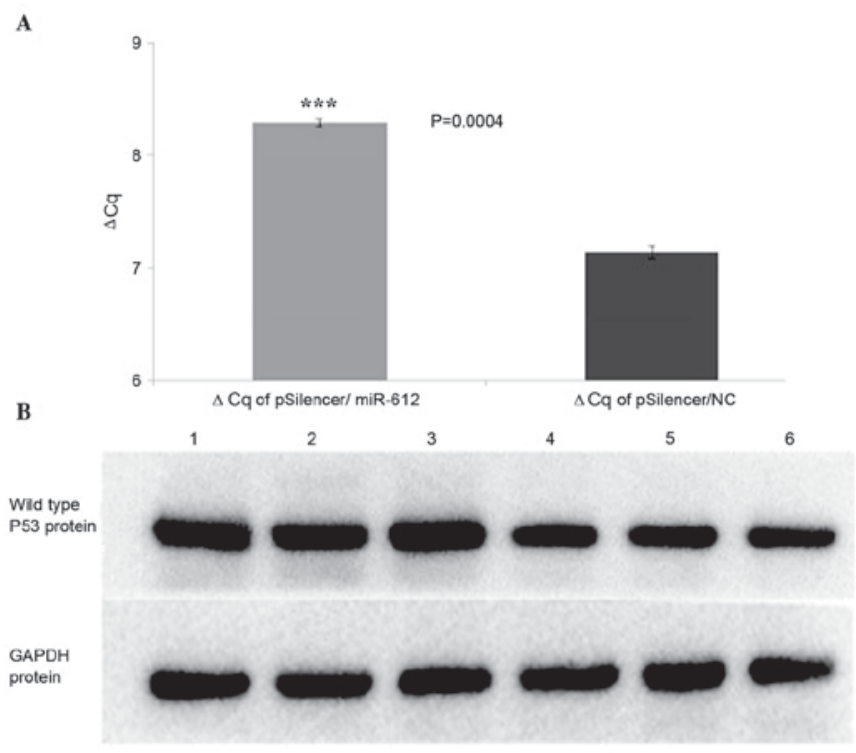

C

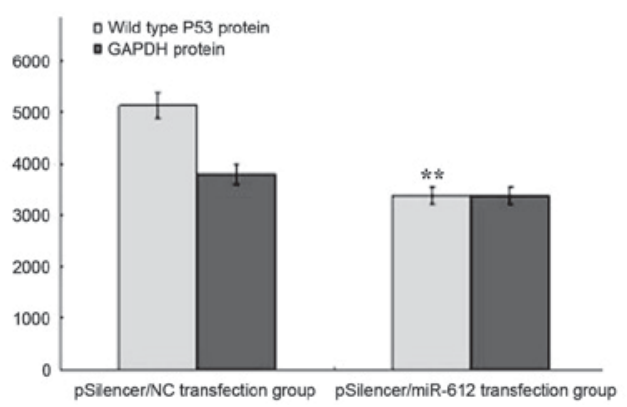

Figure 8. (A) Reverse transcription-quantitative polymerase chain reaction analysis and (B) western blotting to validate the mRNA and protein expression of P53 in the pSilencer/miR-612 plasmid and the negative control pSilencer/NC plasmid transfected EC109 cells. (A) The TP53 mRNA was significantly reduced in the cells that were infected by miR-612 expressed plasmids compared to the negative controls (2.2-fold less). (C) Compared to the control group, the TP53 protein expression in pSilencer/miR-612 transfection group was significantly reduced by $34.0 \%$. Data are presented as means \pm standard deviation. ${ }^{* * *} \mathrm{P}<0.01$ and ${ }^{* * * *} \mathrm{P}<0.001$ vs. pSilencer/NC group. miR, microRNA; NC, negative control.

that miR-612 suppresses the invasive-metastatic cascade in hepatocellular carcinoma. They revealed that miR-612 has inhibitory effects on cell proliferation, migration, invasion and metastasis of hepatocellular carcinoma. This is opposite to what the present study discovered. From multiple target prediction and subsequent experiments, it was identified that miR-612 has an inhibitory action on mRNA and protein expression of TP53. In the authors' 'blast' analysis, the miR-612 sequence at $5^{\prime}$ end matches with the $3^{\prime}$ UTR of TP53. The current fluorescent reporter plasmid experiment indicated that miR-612 reduced the protein expression of theTP53 3'UTR reporter plasmid but has no effect on P53 3'UTR with the mutated reporter plasmid, which clearly presents the specific binding of miR-612 with TP53. The present data demonstrated that the inhibition of miR-612 significantly reduced EC109 cell invasion and migration abilities and further demonstrated the promoting role of miR-612 in ESCC progression and metastasis.

The role of miR-612 is likely more broad. In the authors' target prediction by TargetScans, Pictar, miRanda and
MirTarget 2, there were 2,800 gene targets predicted by at least three prediction tools. Gene Ontology analysis on these genes suggests these genes are involved in several important cancer related functions/pathways such as cell growth regulation, differentiation, apoptosis, cell-cell adhesion and cell-matrix adhesion, and cell invasion and migration (25-30). The genes that participate in cell apoptosis and proliferation include P53, MAPK, WNT and BCL2, and the genes that promote angiogenesis include TGF $\alpha$ and TGF $\beta$.

P53 is a tumor suppressor and serves an important role in promoting cell apoptosis. P53 may be wild type or mutant type. The former acts as a tumor suppressor and participates in many cell activities, such as gene transcription, DNA repair, cell cycle and apoptosis regulation, and cell proliferation and differentiation. The wild type is the key for tumor sensitivity to radiation and chemotherapy, induction to formation of TNF and IL-6 inflammatory agents, and inhibition of angiogenesis in tumors. The mutant P53 may lead to unchecked cell proliferation and tumor development. $~ 50 \%$ tumors harbor P53 point mutations, gene loss or loss of function (23). The current data indicated that wild type TP53 is reduced in primary esophageal SCC and even more in the tumors with lymph node metastasis. As demonstrated, this change is likely mediated through the action of miR-612, thus promoting esophageal cancer invasion and metastasis. The presented results provide a solid foundation for further investigation of the therapeutic potential of miR-612.

\section{Acknowledgements}

The present work was supported by the Natural Scientific Foundation of Shandong (grant no. ZR2012HM085) and the National Natural Science Foundation of China (grant no. 81272420) and Shandong Project of Medicine and Health Technology Development (grant no. 2015WSB04049). The authors would like to thank the patients and investigators for their participation in the current study. They would also like to thank Zhifu Sun and Lei Fang for their technical and language-editing assistance.

\section{References}

1. Enzinger PC and Mayer RJ: Esophageal cancer. N Engl J Med 349: 2241-2252, 2003.

2. Li D: The molecular epidemiologic research of esophageal carcinoma in China. Chin J Epidemiology 24: 939-943, 2003.

3. Furihata T, Sakai T, Kawamata H, Omotehara F, Shinagawa Y, Imura J, Ueda Y, Kubota K and Fujimori T: A new in vivo model for studying invasion and metastasis of esophageal squamous cell carcinoma. Int J Oncol 19: 903-907, 2001.

4. Nelson KM and Weiss GJ: MicroRNAs and cancer: Past, present, and potential future. Mol Cancer Ther 7: 3655-3660, 2008.

5. Latronico MV, Catalucci D and Condorelli G: MicroRNA and cardiac pathologies. Physiol Genomics 34: 239-242, 2008.

6. Feber A, Xi L, Luketich JD, Pennathur A, Landreneau RJ, Wu M, Swanson SJ, Godfrey TE and Litle VR: MicroRNA expression profiles of esophageal cancer. J Thorac Cardiovasc Surg 135: 255-260, 2008.

7. Guo Y, Chen Z, Zhang L, Zhou F, Shi S, Feng X, Li B, Meng X, Ma X, Luo M, et al: Distinctive microRNA profiles relating to patient survival in esophageal squamous cell carcinoma. Cancer Res 68: 26-33, 2008.

8. Ogawa R, Ishiguro H, Kuwabara Y, Kimura M, Mitsui A, Katada T, Harata K, Tanaka T and Fujii Y: Expression profiling of micro-RNAs in human esophageal squamous cell carcinoma using RT-PCR. Med Mol Morphol 42: 102-109, 2009. 
9. Tibshirani R, Hastie T, Narasimhan B and Chu G: Diagnosis of multiple cancer types by shrunken centroids of gene expression. Proc Natl Acad Sci USA 99: 6567-6572, 2002.

10. Livak KJ and Schmittgen TD: Analysis of relative gene expression data using real-time quantitative PCR and the 2(-Delta Delta C(T)) method. Methods 25: 402-408, 2001.

11. Bass AJ and Meyerson M: Genome-wide association study in esophageal squamous cell carcinoma. Gastroenterology 137: 1573-1576, 2009.

12. Wang LD, Zhou FY, Li XM, Sun LD, Song X, Jin Y, Li JM, Kong GQ, Qi H, Cui J, et al: Genome-wide association study of esophageal squamous cell carcinoma in Chinese subjects identifies susceptibility loci at PLCE1 and C20orf54. Nat Genet 42: 759-763, 2010

13. Wu C, Hu Z, He Z, Jia W, Wang F, Zhou Y, Liu Z, Zhan Q, Liu Y, $\mathrm{Yu} \mathrm{D}$, et al: Genome-wide association study identifies three new susceptibility loci for esophageal squamous-cell carcinoma in Chinese populations. Nat Genet 43: 679-684, 2011.

14. Wu C, Li D, Jia W, Hu Z, Zhou Y, Yu D, Tong T, Wang M, Lin D, Qiao Y, et al: Genome-wide association study identifies common variants in SLC39A6 associated with length of survival in esophageal squamous-cell carcinoma. Nat Genet 45: 632-638, 2013.

15. Shimada H, Ochiai T and Nomura F; Japan p53 Antibody Research Group: Titration of serum P53 antibodies in 1,085 patients with various types of malignant tumors: A multiinstitutional analysis by the Japan P53 antibody research group. Cancer 97: 682-689, 2003.

16. Gomes NP and Espinosa JM: Gene-specific repression of the p53 targer gene PUMA via intragenic CTCF-Cohesin binding. Genes Dev 24: 1022-1034, 2010

17. Le MT, Teh C, Shyh-Chang N, Xie H, Zhou B, Korzh V, Lodish HF and Lim B: Micro RNA-125b is anovel negative regulator of p53. Genes Dev 23: 862-876, 2009.

18. Calin GA, Sevignani C, Dumitru CD, Hyslop T, Noch E, Yendamuri S, Shimizu M, Rattan S, Bullrich F, Negrini M and Croce CM: Human microRNA genes are frequently located at fragile sites and genomic regions involved in cancers. Proc Nat Acad Sci USA 101: 2999-3004, 2004

19. Luthra R, Singh RR, Luthra MG, Li YX, Hannah C, Romans AM, Barkoh BA, Chen SS, Ensor J, Maru DM, et al: MicroRNA-196a targets annexin A1: A microRNA-mediated mechanism of annexin A1 downregulation in cancers. Oncogene 27: 6667-6678, 2008.
20. Lee KH, Goan YG, Hsiao M, Lee CH, Jian SH, Lin JT, Chen YL and Lu PJ: MicroRNA-373 (miR-373) post-transcriptionally regulates large tumor suppressor, homolog 2 (LATS2) and stimulates proliferation in human esophageal cancer. Exp Cell Res 315: 2529-2538, 2009.

21. Kurashige J, Kamohara H, Watanabe M, Tanaka Y, Kinoshita K, Saito S, Hiyoshi Y, Iwatsuki M, Baba Y, Baba H, et al: Serum microRNA-21 is a novel biomarker in patients with esophageal squamous cell carcinoma. J Surg Oncol 106: 188-192, 2012.

22. Tian Y, Luo A, Cai Y, Su Q, Ding F, Chen H and Liu Z MicroRNA-10b promotes migration and invasion through KLF4 in human esophageal cancer cell lines. J Biol Chem 285: 7986-7994, 2010

23. Matsushima K, Isomoto H, Kohno S and Nakao K: MicroRNAs and esophageal squamous cell carcinoma. Digestion 82: 138-144, 2010.

24. Wu BL, Xu LY, Du ZP, Liao LD, Zhang HF, Huang Q, Fang GQ and Li EM: MiRNA profile in esophageal squamous cell carcinoma: Downregulation of miR-143 and miR-145. World J Gastroenterol 17: 79-88, 2011.

25. Tian S, Huang S, Wu S, Guo W, Li J and He X: MicroRNA-1285 inhibits the expression of $\mathrm{p} 53$ by directly targeting its 31 untranslated region. Biochem Biophys Res Commun 396: 435-439, 2010.

26. Tao ZH, Wan JL, Zeng LY, Xie L, Sun HC, Qin LX, Wang L, Zhou J, Ren ZG, Li YX, et al: miR-612 suppresses the invasive-metastatic cascade in hepatocellular carcinoma. J Exp Med 210: 789-803, 2013.

27. Landi D, Gemignani F, Naccarati A, Pardini B, Vodicka P, Vodickova L, Novotny J, Försti A, Hemminki K, Canzian F and Landi S: Polymorphisms within micro-RNA-binding sites and risk of sporadic colorectal cancer. Carcinogenesis 29: 579-584, 2008.

28. Balaguer F, Moreira L, Lozano JJ, Link A, Ramirez G, Shen Y, Cuatrecasas M, Arnold M, Meltzer SJ, Syngal S, et al: Colorectal cancers with microsatellite instability display unique miRNA profiles. Clin Cancer Res 17: 6239-6249, 2011.

29. Kim HK, Prokunina-Olsson L and Chanock SJ: Common genetic variants in miR-1206 (8q24.2) and miR-612 (11q13.3) affect biogenesis of mature miRNA forms. PLoS One 10: e47454, 2012.

30. Tang J, Tao ZH, Wen D, Wan JL, Liu DL, Zhang S, Cui JF, Sun HC, Wang L, Zhou J, et al: MiR-612 suppresses the stemness of liver cancer via Wnt/ $\beta$-catenin signaling. Biochem Biophys Res Commun 447: 210-215, 2014. 